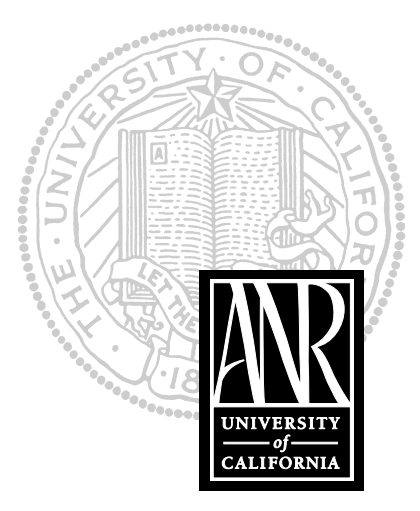

UNIVERSITY OF CALIFORNIA

Division of Agriculture and Natural Resources

http://anrcatalog.ucdavis.edu
PUBLICATION $\mathbf{8 0 1 2}$

\section{Herbicide Resistance: Definition and Management Strategies}

TIMOTHY S. PRATHER, IPM Weed Ecologist, UC Kearney Agricultural Center, Parlier; JOSEPH M. DITOMASO, Cooperative Extension Weed Specialist, UC Davis; and JODIE S. HOLT, Professor, Department of Botany and Plant Sciences, UC Riverside

H erbicide resistance is the inherited ability of a plant to survive and reproduce following exposure to a dose of herbicide that would normally be lethal to the wild type. In a plant, resistance may occur naturally due to selection or it may be induced through such techniques as genetic engineering. Resistance may occur in plants as the result of random and infrequent mutations; there has been no evidence to date that demonstrates herbicide-induced mutation. Through selection, where the herbicide is the selection pressure, susceptible plants are killed while herbicideresistant plants survive to reproduce without competition from susceptible plants. If the herbicide is continually used, resistant plants successfully reproduce and become dominant in the population. The appearance of herbicide resistance in a population is an example of rapid weed evolution (Figure 1).

Research on early cases of herbicide resistance showed that resistant plants were found infrequently in weed populations before use of the herbicide. In some cases this was because the resistant plant was not as fit (i.e., as likely to survive and produce seed) as other plants in the population and therefore would not persist in large numbers. Recent research, however, has shown that in some cases resistance does

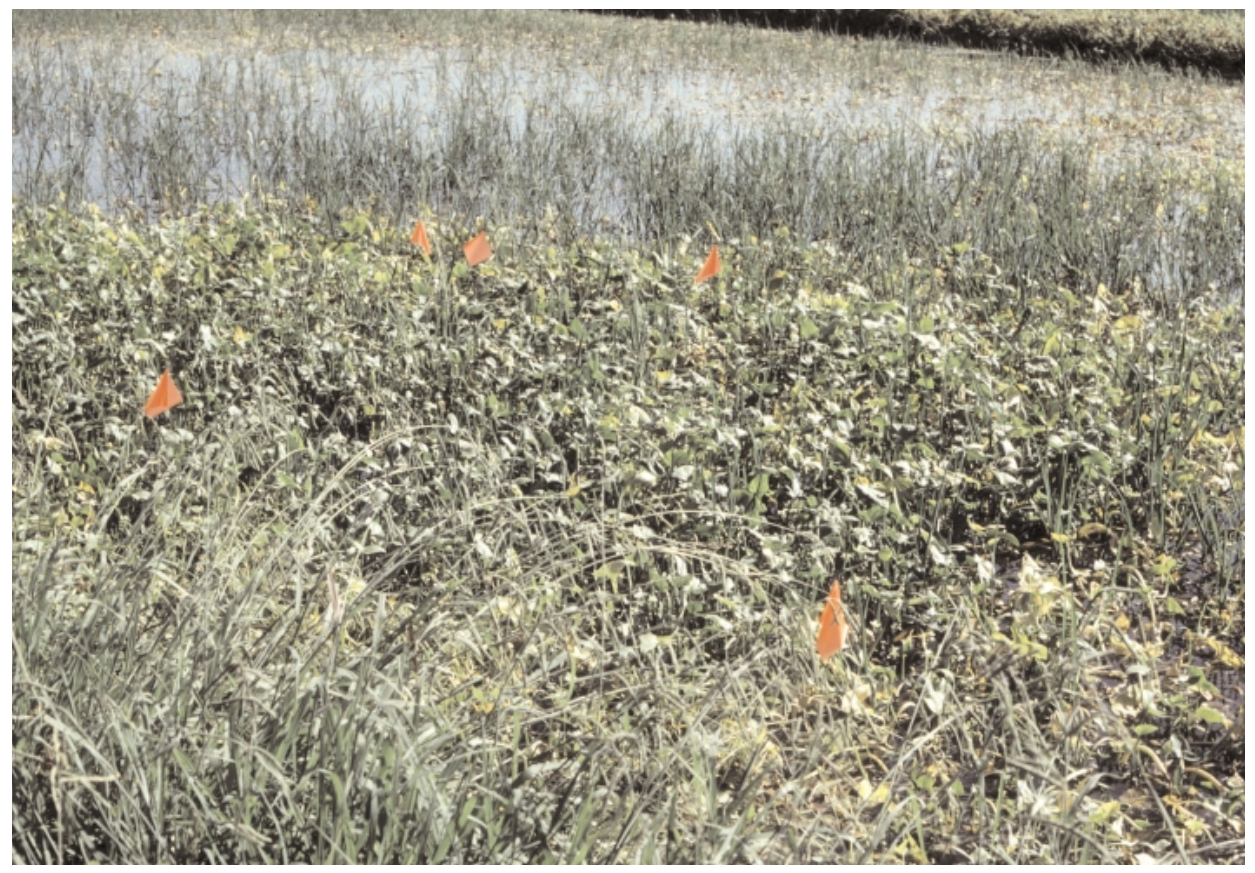

Figure 1. Only a few years after sulfonylurea herbicides were registered for weed control in rice, herbicide resistance had reached detectable levels. 
not come at a cost (e.g., a reduction in fitness), so resistant plants may be just as capable of surviving to reproduce as are susceptible plants. These results suggest that the frequency of resistant plants in a population might be high even before the herbicide selection pressure is applied.

Herbicides are active at one or more target sites within a plant. Target sites are enzymes, proteins, or other places in the plant where herbicides bind and thereby disrupt normal plant functions. One example is an enzyme-acetolactate synthase (ALS, also called acetohydroxy acid synthase [AHAS]) — that is involved in making branched-chain amino acids. Some classes of herbicide (e.g., sulfonylurea, imidazolinone, and pyrimidinyloxybenzoates) bind to the enzyme, causing dysfunction of the enzyme and reducing the synthesis of certain amino acids that are necessary for protein synthesis. These ALS herbicides differ in chemical structure but are active at the same target site. Plants resistant to ALS herbicides have altered acetolactate synthase that does not bind the herbicide. Often, a resistant weed that has been selected by pressure from one herbicide will be resistant to all herbicides that act on that herbicide's target site. When a plant expressing resistance to a herbicide also demonstrates resistance to other herbicides that target the same plant process even though the plant has not been exposed to the other herbicides, the resistance is termed cross-resistance. For example, a population of yellow starthistle in Washington State evolved resistance to Picloram, a picolinic acid herbicide. When that population was subsequently exposed to Clopyralid, another picolinic acid herbicide, it also expressed resistance.

Most cases of herbicide resistance in weeds involve a single mutation or modification in some function so that the weed is resistant or cross-resistant. Rarely does a single plant express resistance to several herbicides that affect different target sites. When a weed that has been exposed to herbicides that attack different target sites expresses resistance to more than one of these herbicides, that is termed multiple resistance. Plants of rigid ryegrass (Lolium rigidum) in Australia have multiple resistance to a number of herbicides in the cyclohexanedione, sulfonylurea, dinitroaniline, triazine, substituted urea, and triazole classes to which the weed has not been exposed. These classes include all of the herbicides currently registered in areas where this weed is a problem. The mechanisms of multiple resistance in rigid ryegrass include changes to the herbicides' sites of action and the detoxification of herbicides by plant enzymes called cytochrome $\mathrm{P}_{450}$ mixed-function oxidases (MFOs). This family of enzymes is similar to those found in many insects resistant to insecticides.

\section{STATUS OF RESISTANCE}

Herbicide resistance was first reported in 1970. Common groundsel (Senecio vulgaris) in a Washington tree nursery was shown to be resistant to herbicides in the triazine chemical class. Since that time, plants of 61 species (42 dicots and 19 monocots) have evolved resistance to the triazine herbicides. Resistance did not evolve in plants as early as it did in insects or fungi due to fundamental differences in the life cycles and genetics of plants, insects, and fungi. The delay in the appearance of resistant weeds is generally attributed to the slower generation time of plants, incomplete selection pressure from most herbicides, soil seed reserves, and the plasticity of weedy plants, all of which keep susceptible individuals in a population and thus delay the evolution of resistance. The appearance of herbicide resistance in plants today is increasing at an exponential rate (Figure 2), mirroring the trends previously seen with insecticide and fungicide resistance. Besides triazine resistance, there are biotypes of 172 weed species expressing resistance to 16 other herbicide classes. 


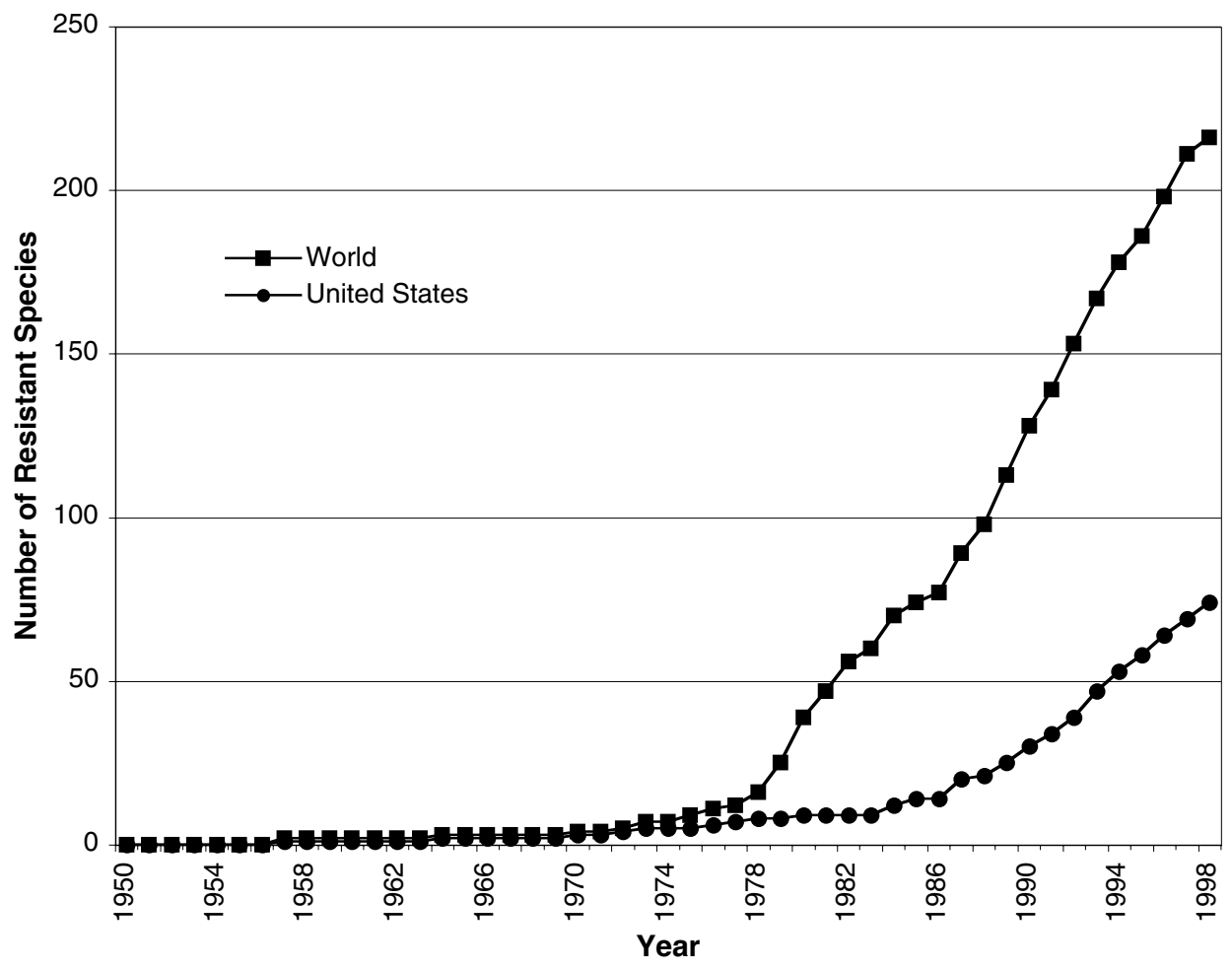

Figure 2. A rapid worldwide increase in herbicide resistance in weeds began in the late 1970 s and continues to the present.

The most common mechanism of action or target site of herbicides, the chemical class, and the number of species with biotypes resistant to each herbicide class are summarized in Table 1.

In California, herbicide resistance today is most widespread among aquatic weeds in rice production (Table 2). Many of these weed species have been selected for resistance to the sulfonylurea herbicide bensulfuron. There has also been one report of triazine resistance as well as one report of sulfonylurea resistance in a noncrop area. A roadside survey conducted in 1995 and 1996 found that resistance to sulfonylurea herbicides was common in Russian thistle (Salsola tragus). Most recently, a rigid ryegrass (Lolium rigidum) biotype exhibited resistance to glyphosate in a northern California orchard. Despite these examples, there are few reports to date of herbicide resistance in California, but the problem is significant in the United States and worldwide (Table 1).

Many current and pending registrations in California, however, involve herbicides that act on branched-chain amino acid synthesis (e.g., Accent, Pursuit, ShadeOut, and Upbeet). The use of herbicides in this group has selected many weed species for resistance in the United States and several in California (Table 2). In addition, a number of genetically engineered crops that are resistant to specific herbicides-such as Roundup Ready cotton and corn-will soon be available in California. Sole reliance on the herbicide to which these crop varieties are resistant will increase the selection pressure on weeds for resistance to the herbicide used. Herbicide-resistant crops will not be an end-all solution to weed problems, and they will not be a useful tool for weed management if used exclusively.

Nationally, an average of six to seven herbicides were registered every year from 1955 to 1975. Since the mid 1970s that number has declined, reaching lows of one to two herbicides per year in the 1980s. Even fewer herbicides are registered for use 
Table 1. Herbicides, their mode of action, and herbicide classes registered for use in California (Resistance has evolved in many groups and is listed in terms of weeds worldwide, in the United States, and in California. Chemical families marked with the same code have been shown to result in cross-resistance among weed species.)

\begin{tabular}{|c|c|c|c|c|c|c|c|}
\hline \multirow[b]{2}{*}{$\begin{array}{l}\text { Resistance } \\
\text { code (HRAC) }\end{array}$} & \multirow[b]{2}{*}{ Mode of action } & \multirow[b]{2}{*}{$\begin{array}{c}\text { Trade } \\
\text { name(s) }\end{array}$} & \multirow[b]{2}{*}{$\begin{array}{c}\text { Common } \\
\text { name }\end{array}$} & \multirow[b]{2}{*}{$\begin{array}{l}\text { Chemical } \\
\text { class }\end{array}$} & \multicolumn{3}{|c|}{$\begin{array}{c}\text { Number of } \\
\text { resistant weed species }\end{array}$} \\
\hline & & & & & $\begin{array}{l}\text { World- } \\
\text { wide }\end{array}$ & $\begin{array}{l}\text { United } \\
\text { States }\end{array}$ & $\begin{array}{l}\text { Cali- } \\
\text { fornia }\end{array}$ \\
\hline$A$ & $\begin{array}{l}\text { Lipid synthesis inhibitors at } \\
\text { acetyl CoA carboxylase } \\
\text { (ACCase) }\end{array}$ & $\begin{array}{l}\text { Hoelon } \\
\text { Whip, Acclaim } \\
\text { Fusilade }\end{array}$ & $\begin{array}{l}\text { Diclofop } \\
\text { Fenoxaprop } \\
\text { Fluazifop }\end{array}$ & $\begin{array}{l}\text { Aryloxy phenoxy } \\
\text { proprionate }\end{array}$ & 18 & 9 & 1 \\
\hline$A$ & $\begin{array}{l}\text { Lipid synthesis inhibitors at } \\
\text { acetyl CoA carboxylase } \\
\text { (ACCase) }\end{array}$ & $\begin{array}{l}\text { Prism, Select } \\
\text { Poast, Vantage }\end{array}$ & $\begin{array}{l}\text { Clethodim } \\
\text { Sethoxydim }\end{array}$ & Cyclohexanedione & 8 & 1 & 0 \\
\hline B & $\begin{array}{l}\text { Branched-chain amino acid } \\
\text { synthesis inhibitors at } \\
\text { acetolactate synthase (ALS); } \\
\text { also called acetohydroxyacid } \\
\text { synthase (AHAS) }\end{array}$ & $\begin{array}{l}\text { Arsenal, Stalker, } \\
\text { Chopper } \\
\text { Pursuit }\end{array}$ & $\begin{array}{l}\text { Imazapyr } \\
\text { Imazethapyr }\end{array}$ & Imidazolinones & 15 & 12 & 0 \\
\hline B & $\begin{array}{l}\text { Branched-chain amino acid } \\
\text { synthesis inhibitors at } \\
\text { acetolactate synthase (ALS); } \\
\text { also called acetohydroxyacid } \\
\text { synthase (AHAS) }\end{array}$ & $\begin{array}{l}\text { Londax } \\
\text { Telar, Glean } \\
\text { Manage, Permit } \\
\text { Accent } \\
\text { Shade-Out, Matrix } \\
\text { Oust } \\
\text { Upbeet }\end{array}$ & $\begin{array}{l}\text { Bensulfuron } \\
\text { Chlorsulfuron } \\
\text { Halosulfuron } \\
\text { Nicosulfuron } \\
\text { Rimsulfuron } \\
\text { Sulfometuron } \\
\text { Triflusulfuron }\end{array}$ & Sulfonylureas & 48 & 17 & 6 \\
\hline B & $\begin{array}{l}\text { Branched-chain amino acid } \\
\text { synthesis inhibitors at } \\
\text { acetolactate synthase (ALS); } \\
\text { also called acetohydroxyacid } \\
\text { synthase (AHAS) }\end{array}$ & Staple & Pyrithiobac* & $\begin{array}{l}\text { Pyrimidinyl- } \\
\text { oxybenzoates }\end{array}$ & 0 & 0 & 0 \\
\hline $\mathrm{C} 1$ & $\begin{array}{l}\text { Photosynthesis inhibitors at } \\
\text { photosystem II }\end{array}$ & $\begin{array}{l}\text { Aatrex and others } \\
\text { Bladex } \\
\text { Pramitol } \\
\text { Caparol and others } \\
\text { Princep }\end{array}$ & $\begin{array}{l}\text { Atrazine } \\
\text { Cyanazine } \\
\text { Prometon } \\
\text { Prometryn } \\
\text { Simazine }\end{array}$ & Triazines & 61 & 17 & 1 \\
\hline $\mathrm{C} 1$ & $\begin{array}{l}\text { Photosynthesis inhibitors at } \\
\text { photosystem II }\end{array}$ & $\begin{array}{l}\text { Velpar, Pronone } \\
\text { Sencor, Lexone }\end{array}$ & $\begin{array}{l}\text { Hexazinone } \\
\text { Metribuzin }\end{array}$ & Triazinones & 3 & 1 & 1 \\
\hline $\mathrm{C} 1$ & $\begin{array}{l}\text { Photosynthesis inhibitors at } \\
\text { photosystem II; same site as } \\
\text { triazines but different binding } \\
\text { behavior }\end{array}$ & $\begin{array}{l}\text { Betanex, Betamix } \\
\text { Betanal, Betamix, } \\
\text { Spin-Aid }\end{array}$ & $\begin{array}{l}\text { Desmedipham } \\
\text { Phenmidipham }\end{array}$ & Phenyl-carbamates & 0 & 0 & 0 \\
\hline $\mathrm{C} 1$ & $\begin{array}{l}\text { Photosynthesis inhibitors at } \\
\text { photosystem Il; same site as } \\
\text { triazines but different binding } \\
\text { behavior }\end{array}$ & $\begin{array}{l}\text { Pyramin } \\
\text { Tough }\end{array}$ & $\begin{array}{l}\text { Pyrazon } \\
\text { Pyridate }\end{array}$ & Pyridazinones & 0 & 0 & 0 \\
\hline $\mathrm{C} 1$ & $\begin{array}{l}\text { Photosynthesis inhibitors at } \\
\text { photosystem II; same site as } \\
\text { triazines but different binding } \\
\text { behavior }\end{array}$ & Hyvar & Bromacil & Uracil & 1 & 1 & 0 \\
\hline $\mathrm{C} 2$ & $\begin{array}{l}\text { Photosynthesis inhibitors at } \\
\text { photosystem II; same site as } \\
\text { triazines but different binding } \\
\text { behavior }\end{array}$ & $\begin{array}{l}\text { Stam, Stampede, } \\
\text { Propanil }\end{array}$ & Propanil & Amide & 2 & 1 & 0 \\
\hline
\end{tabular}


Table 1 (continued). Herbicides, their mode of action, and herbicide classes registered for use in California (Resistance has evolved in many groups and is listed in terms of weeds worldwide, in the United States, and in California. Chemical families marked with the same code have been shown to result in cross-resistance among weed species.)

\begin{tabular}{|c|c|c|c|c|c|c|c|}
\hline \multirow[b]{2}{*}{$\begin{array}{l}\text { Resistance } \\
\text { code (HRAC) }\end{array}$} & \multirow[b]{2}{*}{ Mode of action } & \multirow[b]{2}{*}{$\begin{array}{c}\text { Trade } \\
\text { name(s) }\end{array}$} & \multirow[b]{2}{*}{$\begin{array}{l}\text { Common } \\
\text { name }\end{array}$} & \multirow[b]{2}{*}{$\begin{array}{l}\text { Chemical } \\
\text { class }\end{array}$} & \multicolumn{3}{|c|}{$\begin{array}{c}\text { Number of } \\
\text { resistant weed species }\end{array}$} \\
\hline & & & & & $\begin{array}{l}\text { World- } \\
\text { wide }\end{array}$ & $\begin{array}{l}\text { United } \\
\text { States }\end{array}$ & $\begin{array}{l}\text { Cali- } \\
\text { fornia }\end{array}$ \\
\hline $\mathrm{C} 2$ & $\begin{array}{l}\text { Photosynthesis inhibitors at } \\
\text { photosystem II; same site as } \\
\text { triazines but different binding } \\
\text { behavior }\end{array}$ & $\begin{array}{l}\text { Karmex and others } \\
\text { Lorox } \\
\text { Spike }\end{array}$ & $\begin{array}{l}\text { Diuron } \\
\text { Linuron } \\
\text { Tebuthiuron }\end{array}$ & Ureas & 17 & 4 & 0 \\
\hline $\mathrm{C} 3$ & $\begin{array}{l}\text { Photosynthesis inhibitors at } \\
\text { photosystem II; same site as } \\
\text { triazines but different binding } \\
\text { behavior }\end{array}$ & Basagran, Lescogran & Bentazon & Benzothiadiazole & 0 & 0 & 0 \\
\hline $\mathrm{C} 3$ & $\begin{array}{l}\text { Photosynthesis inhibitors at } \\
\text { photosystem II; same site as } \\
\text { triazines but different binding } \\
\text { behavior }\end{array}$ & Buctril, Moxy & Bromoxynil & Nitriles & 1 & 1 & 0 \\
\hline $\mathrm{D}$ & $\begin{array}{l}\text { Photosynthesis inhibitor; } \\
\text { electron diversion at } \\
\text { photosystem I }\end{array}$ & $\begin{array}{l}\text { Reward, Diquat } \\
\text { Gromoxone, Cyclone, } \\
\text { Starfire }\end{array}$ & $\begin{array}{l}\text { Diquat } \\
\text { Paraquat }\end{array}$ & Bipyriduliums & 25 & 2 & 0 \\
\hline$E$ & $\begin{array}{l}\text { Free radical generator; inhibitor } \\
\text { of protoporphrinogen } \\
\text { oxidase (PPO) }\end{array}$ & Goal & Oxyfluorfen & Diphenyl ether & 0 & 0 & 0 \\
\hline $\mathrm{E}$ & $\begin{array}{l}\text { Free radical generator; inhibitor } \\
\text { of protoporphrinogen } \\
\text { oxidase (PPO) }\end{array}$ & Ronstar & Oxadiazon & Oxadiazole & 0 & 0 & 0 \\
\hline $\mathrm{E}$ & $\begin{array}{l}\text { Free radical generator; inhibitor } \\
\text { of protoporphrinogen } \\
\text { oxidase (PPO) }\end{array}$ & Milestone & Azafenidn & Triazolopyridinone & 0 & 0 & 0 \\
\hline F1 & $\begin{array}{l}\text { Bleaching; inhibitor of } \\
\text { carotenoid synthesis at } \\
\text { phytoene desaturase (PDS) }\end{array}$ & $\begin{array}{l}\text { Predict, Solicam, } \\
\text { Zorial }\end{array}$ & Norflurazon & Pyridazinone & 0 & 0 & 0 \\
\hline F1 & $\begin{array}{l}\text { Bleaching; inhibitor of } \\
\text { carotenoid synthesis at } \\
\text { phytoene desaturase (PDS) }\end{array}$ & Sonar & Fluridone & $\begin{array}{l}\text { Unclassified } \\
\text { herbicide }\end{array}$ & 0 & 0 & 0 \\
\hline G & $\begin{array}{l}\text { Aromatic amino acid inhibitor } \\
\text { at EPSP synthase }\end{array}$ & $\begin{array}{l}\text { Roundup, Rodeo, } \\
\text { Accord }\end{array}$ & Glyphosate & Glycine & 2 & 1 & 1 \\
\hline $\mathrm{H}$ & Glutamine synthesis inhibitor & Finale, Liberty, Rely & Glufosinate & Phosphinic acid & 0 & 0 & 0 \\
\hline K1 & $\begin{array}{l}\text { Mitotic disruptors; microtubule } \\
\text { assembly inhibitors }\end{array}$ & $\begin{array}{l}\text { Balan } \\
\text { Sonalan, Curbit } \\
\text { Surflan } \\
\text { Prowl, Pendulum } \\
\text { Barricade, } \\
\text { Endurance, Factor } \\
\text { Treflan }\end{array}$ & $\begin{array}{l}\text { Benefin } \\
\text { Ethalfluralin } \\
\text { Oryzalin } \\
\text { Pendimethalin } \\
\text { Prodiamine } \\
\text { Trifluralin }\end{array}$ & Dinitroanilines & 9 & 5 & 0 \\
\hline $\mathrm{K} 1$ & $\begin{array}{l}\text { Mitotic disruptors; microtubule } \\
\text { assembly inhibitors }\end{array}$ & $\begin{array}{l}\text { Dimension } \\
\text { Visor }\end{array}$ & $\begin{array}{l}\text { Dithiopyr } \\
\text { Thiazopyr }\end{array}$ & Pyridazine & 1 & 0 & 0 \\
\hline
\end{tabular}


Table 1 (continued). Herbicides, their mode of action, and herbicide classes registered for use in California (Resistance has evolved in many groups and is listed in terms of weeds worldwide, in the United States, and in California. Chemical families marked with the same code have been shown to result in cross-resistance among weed species.)

\begin{tabular}{|c|c|c|c|c|c|c|c|}
\hline \multirow[b]{2}{*}{$\begin{array}{l}\text { Resistance } \\
\text { code (HRAC) }\end{array}$} & \multirow[b]{2}{*}{ Mode of action } & \multirow[b]{2}{*}{$\begin{array}{c}\text { Trade } \\
\text { name(s) }\end{array}$} & \multirow[b]{2}{*}{$\begin{array}{l}\text { Common } \\
\text { name }\end{array}$} & \multirow[b]{2}{*}{$\begin{array}{l}\text { Chemical } \\
\text { class }\end{array}$} & \multicolumn{3}{|c|}{$\begin{array}{c}\text { Number of } \\
\text { resistant weed species }\end{array}$} \\
\hline & & & & & $\begin{array}{l}\text { World- } \\
\text { wide }\end{array}$ & $\begin{array}{l}\text { United } \\
\text { States }\end{array}$ & $\begin{array}{l}\text { Cali- } \\
\text { fornia }\end{array}$ \\
\hline K3 & $\begin{array}{l}\text { Mitotic disruptor; microtubule } \\
\text { assembly inhibitors; different } \\
\text { site than dinitroanilines }\end{array}$ & Kerb & Pronamide & Benzamide & 1 & 1 & 0 \\
\hline K3 & $\begin{array}{l}\text { Cell division inhibitor; } \\
\text { site unknown }\end{array}$ & Devrinol & Napropamide & Acetamide & 0 & 0 & 0 \\
\hline K3 & $\begin{array}{l}\text { Lipid synthesis inhibitors; } \\
\text { not ACCase }\end{array}$ & $\begin{array}{l}\text { Lasso, Partner } \\
\text { Dual }\end{array}$ & $\begin{array}{l}\text { Alachlor } \\
\text { Metolach }\end{array}$ & Chloroacetamide & 3 & 0 & 0 \\
\hline L & Cell wall synthesis inhibitor & Gallery & Isoxaben & Benzamide & 0 & 0 & 0 \\
\hline $\mathrm{L}$ & $\begin{array}{l}\text { Cell wall (cellulose) synthesis } \\
\text { inhibitor }\end{array}$ & Casoron, Norosac & Dichlobenil & Nitrile & 0 & 0 & 0 \\
\hline $\mathrm{N}$ & $\begin{array}{l}\text { Lipid synthesis inhibitors; } \\
\text { not ACCase }\end{array}$ & $\begin{array}{l}\text { Sutan } \\
\text { Ro-Neet } \\
\text { Eptam, Eradicane } \\
\text { Ordram } \\
\text { Tillam } \\
\text { Bolero }\end{array}$ & $\begin{array}{l}\text { Butylate } \\
\text { Cycloate } \\
\text { EPTC } \\
\text { Molinate } \\
\text { Pebulate } \\
\text { Thiobencarb }\end{array}$ & Thiocarbamates & 3 & 2 & 1 \\
\hline $\mathrm{N}$ & $\begin{array}{l}\text { Lipid synthesis inhibitors; } \\
\text { not ACCase }\end{array}$ & $\begin{array}{l}\text { Prefar, Betasan } \\
\text { Dual }\end{array}$ & $\begin{array}{l}\text { Bensulide } \\
\text { Metolachlor }\end{array}$ & $\begin{array}{l}\text { Phosphorodithioates } \\
\text { Acetamide }\end{array}$ & 0 & 0 & 0 \\
\hline $\mathrm{N}$ & Fatty acid synthesis inhibitor & Norton, Prograss & Ethofumesate & Benzofuran & 1 & 1 & 0 \\
\hline 0 & $\begin{array}{l}\text { Growth regulators; synthetic } \\
\text { auxins (action similar to } \\
\text { indoleacetic acid) }\end{array}$ & $\begin{array}{l}\text { Banvel, Vanquish, } \\
\text { Clarity }\end{array}$ & Dicamba & Benzoic acids & 2 & 1 & 0 \\
\hline 0 & $\begin{array}{l}\text { Growth regulators; synthetic } \\
\text { auxins (action similar to } \\
\text { indoleacetic acid) }\end{array}$ & $\begin{array}{l}\text { Several } \\
\text { Several } \\
\text { Several }\end{array}$ & $\begin{array}{l}\text { 2.4-D } \\
\text { MCPA } \\
\text { Mecoprop } \\
\text { (MCPP) }\end{array}$ & $\begin{array}{l}\text { Phenoxy carboxylic } \\
\text { acids }\end{array}$ & 19 & 6 & 0 \\
\hline 0 & $\begin{array}{l}\text { Growth regulators; synthetic } \\
\text { auxins (action similar to } \\
\text { indoleacetic acid) }\end{array}$ & $\begin{array}{l}\text { Transline, Stinger } \\
\text { Garlon, Remedy, } \\
\text { Pathfinder, } \\
\text { Grandstand, Turflon }\end{array}$ & $\begin{array}{l}\text { Clopyralid } \\
\text { Triclopyr }\end{array}$ & Picolinic acids & 1 & 1 & 0 \\
\hline Z & Membrane disruptors & $\begin{array}{l}\text { DSMA and others } \\
\text { Several }\end{array}$ & $\begin{array}{l}\text { DSMA } \\
\text { MSMA }\end{array}$ & Organic arsenicals & 1 & 1 & 0 \\
\hline Z & $\begin{array}{l}\text { Rapid membrane destruction; } \\
\text { site unknown }\end{array}$ & Scythe & $\begin{array}{l}\text { Pelargonic } \\
\text { acid }\end{array}$ & $\begin{array}{l}\text { Unclassified } \\
\text { herbicide }\end{array}$ & 0 & 0 & 0 \\
\hline Z & Unknown & Avenge & Difenzoquat & Pyrazolium salt & 1 & 1 & 1 \\
\hline Z & Unknown & Metam, Vapam & Metham & Dithiocarbamate & 0 & 0 & 0 \\
\hline none & Unknown & $\begin{array}{l}\text { Aquathol, } \\
\text { Hydrothal, and } \\
\text { others }\end{array}$ & Endothall & $\begin{array}{l}\text { Disodium salt of } \\
\text { methanearsonate }\end{array}$ & 0 & 0 & 0 \\
\hline none & Unknown & $\begin{array}{l}\text { Several } \\
\text { Barespot Monobor- } \\
\text { Chlorate }\end{array}$ & $\begin{array}{l}\text { Copper sulfate } \\
\text { and chelate } \\
\text { Sodium chlorate } \\
\text { and metaborate }\end{array}$ & Inorganics & 0 & 0 & 0 \\
\hline
\end{tabular}


Table 2. Herbicide-resistant weeds in California

\begin{tabular}{|c|c|c|c|c|}
\hline Species & Common name & Area & $\begin{array}{c}\text { Year } \\
\text { reported }\end{array}$ & $\begin{array}{c}\text { Chemical class } \\
\text { (herbicide) }\end{array}$ \\
\hline Senecio vulgaris & Common groundsel & Orchard & 1981 & $\begin{array}{l}\text { Triazine } \\
\text { (atrazine) }\end{array}$ \\
\hline Lolium perenne & Perennial ryegrass & Roadside & 1989 & $\begin{array}{l}\text { Sulfonylurea } \\
\text { (sulfometuron) }\end{array}$ \\
\hline Cyperus difformis & $\begin{array}{l}\text { Smallflower } \\
\text { umbrella sedge }\end{array}$ & Rice & 1993 & $\begin{array}{l}\text { Sulfonylurea } \\
\text { (bensulfuron) }\end{array}$ \\
\hline $\begin{array}{l}\text { Sagittaria } \\
\text { montevidensis }\end{array}$ & $\begin{array}{l}\text { California } \\
\text { arrowhead }\end{array}$ & Rice & 1993 & $\begin{array}{l}\text { Sulfonylurea } \\
\text { (bensulfuron) }\end{array}$ \\
\hline Salsola tragus & Russian thistle & Roadside & 1994 & $\begin{array}{l}\text { Sulfonylurea } \\
\text { (sulfometuron) }\end{array}$ \\
\hline Ammania auriculata & Redstem & Rice & 1997 & $\begin{array}{l}\text { Sulfonylurea } \\
\text { (bensulfuron) }\end{array}$ \\
\hline Scirpus mucronatus & Ricefield bulrush & Rice & 1997 & $\begin{array}{l}\text { Sulfonylurea } \\
\text { (bensulfuron) }\end{array}$ \\
\hline $\begin{array}{l}\text { Echinochloa } \\
\text { phyllopogon }\end{array}$ & Late watergrass & Rice & 1998 & $\begin{array}{l}\text { Thiocarbamate } \\
\text { (thiobencarb) }\end{array}$ \\
\hline $\begin{array}{l}\text { Echinochloa } \\
\text { phyllopogon }\end{array}$ & Late watergrass & Rice & 1998 & $\begin{array}{l}\text { Aryloxyphenoxy } \\
\text { propionic acid } \\
\text { (fenoxaprop) }\end{array}$ \\
\hline $\begin{array}{l}\text { Echinochloa } \\
\text { crus-galli }\end{array}$ & Barnyardgrass & Cotton & 1999 & $\begin{array}{l}\text { Dinitroaniline } \\
\text { (trifluralin) }\end{array}$ \\
\hline Avena fatua & Wild oat & $\begin{array}{l}\text { Barley, } \\
\text { Wheat }\end{array}$ & 1996 & $\begin{array}{l}\text { Pyrazolium salt } \\
\text { (difenzoquat) }\end{array}$ \\
\hline Lolium rigidum & Rigid ryegrass & Orchard & 1998 & $\begin{array}{l}\text { Substituted } \\
\text { amino acid } \\
\text { (glyphosate) }\end{array}$ \\
\hline
\end{tabular}

in California. Re-registration requirements for pesticides have also resulted in the loss of herbicide registrations in many crops. With few new herbicide registrations and a loss of existing compounds, the potential for repeated use of the limited number of herbicides available is increasing, and that increases the potential for selecting resistance in weeds. Weed control programs should include strategies that reduce the likelihood of selection for herbicide-resistant weeds and conserve existing chemical tools.

\section{CONTRIBUTING FACTORS}

Evolution and natural selection are the processes that have led to the plant species found around the world today. Many plants, particularly weeds, contain a tremendous amount of genetic variation that allows them to survive under a variety of environmental conditions. Most herbicides affect a single specific site of action, and that site is usually under the control of a single gene, or at most a few genes. With a single gene mutation, even minor changes in gene expression can confer resistance by modifying the site where a herbicide has its toxic effect: the site of action. The evolution of a resistant population in a species comes about in response to selection pressure imposed by that herbicide or by another herbicide that shares the same site of action. When a herbicide exerts selection pressure on a population, plants possessing the resistance trait have a distinct advantage. Unlike susceptible plants, resis- 
tant plants will survive and reproduce. Continuous herbicide exposure maintains the selection pressure and thereby rapidly increases the number of resistant plants.

Weeds possess traits that promote the evolution of resistance. A high rate of seed production with most seed germinating within a year can accelerate the evolution of resistance. When susceptible plants are removed from the population by the herbicide, prolific seed production by resistant plants rapidly shifts the population toward resistance. High seed production coupled with genetic variation increases the probability that resistance will evolve. Perennial weeds, particularly those with vegetative reproductive tissues, are less likely to evolve resistance than are weeds with an annual life cycle that produce abundant seeds, since generally there is less genetic diversity in perennial weeds that reproduce vegetatively and fewer opportunities for new mutations to be transferred to offspring via seeds. The most common weed genera that contain herbicide-resistant populations are listed in Table 3. All of these genera are dominated by annual species.

Table 3. Weed genera with the greatest number of resistant species worldwide and in California

\begin{tabular}{llcc}
\hline & & \multicolumn{2}{c}{ Number of occurrences } \\
\cline { 3 - 4 } Genus & Common name & Worldwide & In California \\
\hline Amaranthus & Pigweed & 42 & 0 \\
Chenopodium & Lambsquarters & 25 & 0 \\
Conyza & Fleabane or horseweed & 22 & 0 \\
Lolium & Ryegrass & 21 & 2 \\
Setaria & Foxtail & 17 & 0 \\
Avena & Wild oat & 15 & 1 \\
Echinochloa & Barnyardgrass or watergrass & 15 & 3 \\
Alopecurus & Blackgrass & 13 & 0 \\
Senecio & Groundsel & 12 & 1 \\
Polygonum & Knotweed or smartweed & 12 & 0 \\
Solanum & Nightshade & 11 & 0 \\
\hline
\end{tabular}

In the absence of herbicide treatment, weeds resistant to the triazine herbicides are not as fit as are susceptible plants of the same species. This is because the efficiency of photosynthesis is reduced in resistant plants by the alteration of a specific photosynthetic protein that is also the herbicide binding site, so conferring resistance. Since resistant plants are less fit, they reproduce at lower rates and consequently represent a smaller fraction of the number of individuals within a population. In contrast, some resistance traits do not have the same fitness cost. In those cases, resistant individuals often represent a larger fraction of a population (Figure 3).

The frequency of the resistance trait within the population is an important factor in determining the rate of selection for resistance among weed species. For example, resistance to triazines evolved after 10 years of continual use of the herbicides. Unlike the triazines, the sulfonylurea herbicides have not been shown to have a significant fitness cost associated with the resistance trait. Resistance to these herbicides took only 4 years to evolve. For weed species with resistance to sulfonylurea herbicides, the initial proportion of resistant plants in a population has been estimated at approximately 1 in 1 million individuals. Thus, if a weed population has a density of 10 plants per $\mathrm{m}^{2}$, one would expect to find one resis- 
tant individual in every 10 hectares (24.7 acres) of infestation. Without multiple control strategies, these resistant individuals are likely to survive long enough to produce resistant seed.

Several factors, such as herbicide characteristics, plant characteristics, weed control practices, and production practices, can increase the probability of selection for herbicide resistance. Herbicide factors that contribute to the potential for resistance include a long soil residual activity, a single target site and specific mode of action, and a high effective kill rate for a wide range of weed species. Herbicides with prolonged soil residual activity exert selection pressure for a longer time period since they will kill most of the susceptible plants that germinate over a growing season. A herbicide with a single target site

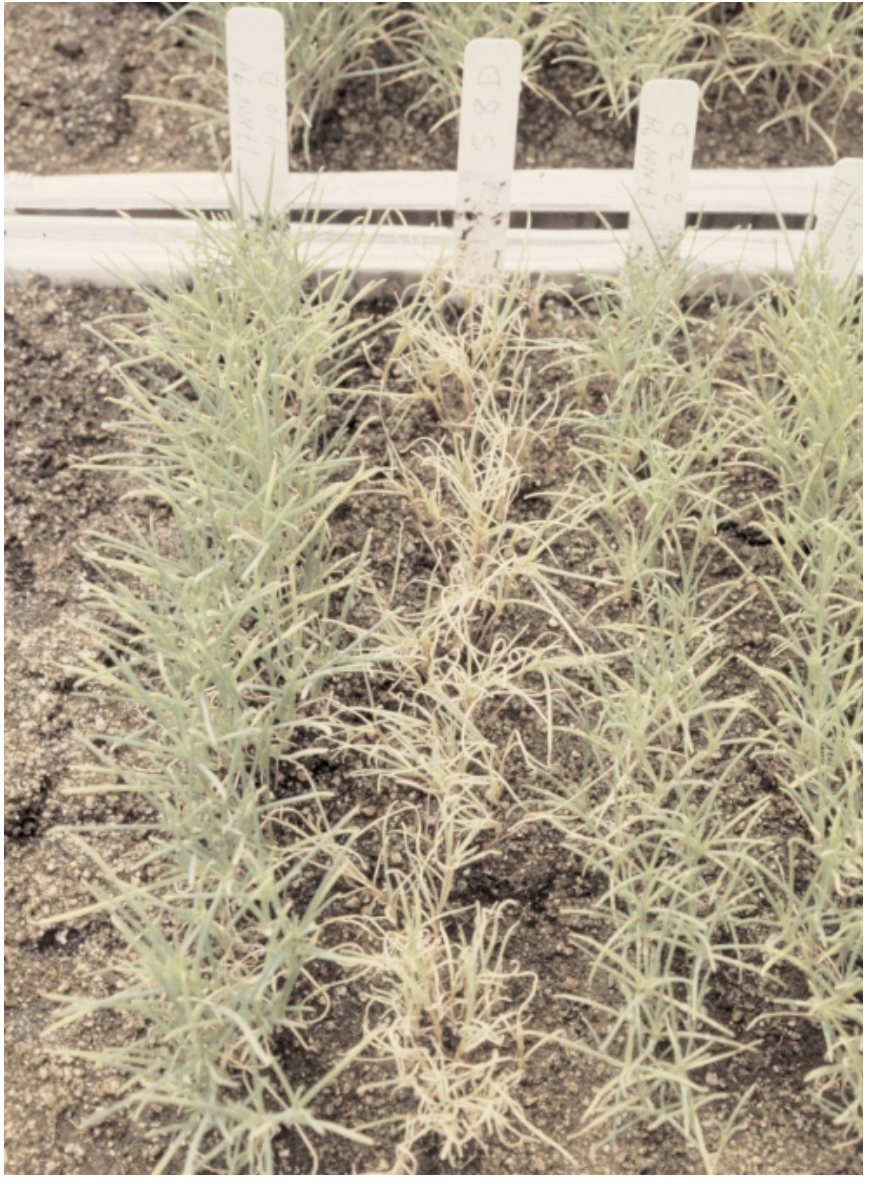

Figure 3. Russian thistle (tumbleweed) along roadsides is resistant to ALS herbicides in areas where these herbicides have been used for several years. Resistant and susceptible plants shown in the picture were sprayed with Telar (chlorsulfuron). controlled by few genes is more likely to encounter plants with mutations for resistance than is a herbicide with several modes of action. A high effective kill rate rapidly depletes susceptible genes from the population, and the result is a rapid increase in resistance among the progeny of a few initial resistant plants.

Although the most common mechanism of herbicide resistance in weeds is an alteration at the site of action, resistance can also result from an enhancement of the plant's ability to metabolize and detoxify the herbicide. This mechanism is not yet widespread in the United States. Like target site changes, selection for enhanced metabolism can also occur in response to repeated applications of the same herbicide or of a group of herbicides that are vulnerable to the same detoxification enzymes. For example, enhanced metabolism is thought to confer resistance to picolinic acid herbicides in yellow starthistle in eastern Washington. Weed biotypes with enhanced metabolism have a much lower level of resistance than weeds expressing resistance through site of action changes. Selection for weeds with enhanced metabolism is more rapid when a herbicide is used continuously at or below the low recommended rate. This allows a gradual increase of the weed biotypes that are more able to metabolize the compound.

The most likely way to cause evolution of resistant weed populations is to exert selection pressure on weeds with the same herbicides over several generations. By using long-soil-residual herbicides, the same herbicide continuously, or a rotation of 
herbicides that target the same site, you apply selection pressure for resistance over several generations.

The continuous planting of the same crop within and between growing seasons reduces your options for rotating to herbicides with different target sites. For example, crop rotation with California rice is difficult, so fields are planted continuously to the same crop. The herbicide bensulfuron (Londax, an ALS [AHAS] inhibiting herbicide) was registered for rice in California in 1989. It was highly effective on most rice weeds. Few alternative control techniques were used in rice, so Londax was used extensively for several years. Resistance evolved quickly, and now at least four weed species are resistant to Londax (Table 2).

The limited number of herbicides registered for many minor crops restricts the grower's ability to rotate among compounds with different sites of action. This often leads to continuous use of one or a few herbicides and increases the probability that herbicide resistance will evolve among weed populations in those fields. Resistance has not yet become a problem in California's minor crop production areas, however. This is probably because of the extensive use of hand labor, cultivation, and frequent rotation among a number of crops for which herbicides with different target sites are registered.

While hand labor and cultivation continue as effective methods for preventing resistance, the herbicide rotation that has accompanied crop rotation may become ineffective as herbicides that target branched-chain amino acid synthesis (sulfonylureas and imidazolinones, ALS [AHAS] inhibitors) are being registered for several of the nationally minor crops grown in California, including tomatoes and sugar beet. In addition, ALS (AHAS) inhibiting herbicides have been registered for cotton, corn, and alfalfa. The risk that weeds will evolve resistance to these herbicides will increase if ALS (AHAS) inhibiting herbicides are used continuously in several crops within a rotation.

The exclusive use of herbicides for weed control can rapidly select for resistance when other control practices such as tillage or hand hoeing are not also used to control herbicide-resistant weeds. In general, nonchemical methods will not select between susceptible and resistant plants, so they should be used whenever possible. Resistance also evolves more quickly in lower-value solid-seeded crops grown on large acreages, since cultivation and hand-weeding of these crops may not be feasible. Farmers who grow crops over large areas tend to rely heavily on herbicides for weed control. A large acreage contains a greater overall number of individual weeds that may contain a resistance trait.

\section{PREVENTION AND MANAGEMENT}

Any management action that reduces the selection pressure for resistance will reduce the rate of resistance evolution. Crop rotation is one of the best tools for preventing resistance. Rotation to another crop allows the grower to use both chemical and non-chemical control methods. Manipulation of planting time, the competitiveness of the crop, cultivation techniques, hand weeding, and applications of herbicides with different target sites all are possible in a crop rotation system. Farmers and Pest Control Advisors (PCAs) in California use many of these methods to control weeds. Probably because of these characteristics of California agriculture, few weed species have evolved herbicide resistance in this state. As highly effective herbicides with the same target site become registered in California in multiple crops of a rotation, however, the risk increases that resistance will evolve. 
Herbicides with different chemistries and trade names but with a target site in common can reduce the effectiveness of herbicide rotation. Some common crop rotations include cotton, corn, tomato, sugarbeet, and alfalfa. All of these crops now have registered herbicides that target the same site (ALS). As noted earlier, biotypes resistant to these herbicides may have no fitness cost associated with resistance and there may be high numbers of resistant individuals in a population. Weed species will evolve resistance rapidly unless farmers rotate to herbicides with different target sites.

Herbicide-resistant crops represent a new technology whose use is increasingly widespread. In many cases, farmers who grow these crops will rely more heavily on a single herbicide. Such a strategy will likely select for weed biotypes that are resistant to that herbicide or mode of action. Tank mixing, rotating herbicides, rotating to varieties without the resistance trait, and integrating non-chemical control options within the weed management program will reduce the potential that weed biotypes will evolve resistance.

The use of short-residual herbicides also reduces selection pressure for herbicide resistance. In addition, tank-mixing of herbicides with different modes of action (Table 1) can inhibit the evolution of resistance, but the combinations used should broaden the spectrum of weeds controlled along with controlling the weed species of major concern. If two herbicides have nearly the same weed control spectrum, you would do better to rotate between them rather than tank-mix the two compounds; little additional control will be achieved by adding the second material. Though unlikely, it is possible in theory that a weed population will simultaneously be selected for resistance to both herbicides.

While weeds have traits that enhance their potential to evolve resistance, they also have traits that reduce that potential. Weed species with seeds that remain dormant in the soil for several years will maintain a population of susceptible plants within the seed bank. By maintaining susceptible plants in the population, a grower can dilute the resistance trait. If there is a fitness cost to resistance, removing the herbicide at some point in the crop rotation cycle will allow competition between the resistant and susceptible plants, further diluting the gene pool for the resistance trait.

Besides the practice of crop rotation, certified seed, equipment sanitation, cultivation, and hand-weeding all impede resistance evolution. Resistance problems usually go undetected until land managers or farmers observe about 30 percent weed control failure for a particular species. If you can identify these resistant weed patches early, before their populations increase, you can employ management practices that prevent their spread. If weed escapes appear in patterns such as distinct strips, or if several species normally controlled by the herbicide are present in these strips, the problem probably is associated with a calibration or application error. However, patches made up of only one escaped species and showing no distinctive pattern may indicate a herbicide-resistant population. Suspicious areas should be brought to the attention of a Farm Advisor or Extension Specialist, especially if weed populations reoccur in subsequent years after use of the same herbicide. 


\section{HOW TO PREVENT OR DELAY HERBICIDE RESISTANCE}

Weed management strategies that discourage the evolution of herbicide resistance should include the following:

- Herbicide rotation

- Use the data in Table 1 to guide your herbicide rotation decisions.

- Crop rotation

- Plant to a crop having a different season of growth.

- Plant to a crop having different registered herbicides (see Table 1).

- Plant to a crop for which there are alternate methods of weed control.

- Monitoring after herbicide application

- Check for weedy patches in patterns consistent with application problems.

- Hand-weed patches that are not in patterns consistent with application problems.

- Non-chemical control techniques

- Cultivate.

- Hand-weed. A 90 percent or greater rate of weed removal reduces the chances that a resistant plant will produce seed.

- Mulching with both synthetic and organic materials.

- Solarize the soil.

- Short-residual herbicides

- Certified seed

- Clean equipment

- Use a power washer or compressed air to remove seeds.

\section{HOW TO MANAGE HERBICIDE-RESISTANT WEEDS}

To keep herbicide-resistant weeds under control, incorporate these strategies into your weed management plan:

- Herbicide rotation

- Use the data in Table 1 to guide your herbicide rotation decisions.

- Fallow tillage

- Close cultivation

- Monitor hand weeding crew to insure more than 90 percent removal of weeds in the crop row.

- Prevention of weed seed spread through use of clean equipment

- Enter the field with resistant plants last.

- Use a power washer or compressed air to remove seeds.

- Monitoring the initial evolution of resistance by recognizing patterns of weed escapes typical of resistant plants

- Watch for small weed patches that appear in the same place in the next crop.

- Watch for weed patches that do not have a regular shape that would indicate a herbicide application problem.

- Control of weeds suspected of herbicide resistance before they can produce seed 


\section{CONCLUSION}

The potential for herbicide resistance should receive serious and thoughtful attention in California. California weed management will change significantly as new herbicides and herbicide-resistant crops are introduced and older herbicides are lost. If we maintain a selection pressure through continuous use of these new tools, herbicide resistance will soon render them ineffective. By adopting proactive management strategies designed to prevent herbicide resistance, we can conserve important weed-control tools. If we ignore resistance-management strategies, it is likely that IPM systems will lose the flexibility necessary to deal with weed problems.

\section{ADDITIONAL RESOURCES}

Anonymous. 1993. Guidelines for managing resistance to ALS/AHAS-inhibiting herbicides. ALS/AHAS Inhibitor Resistance Working Group.

Anonymous. 1993. Londax resistance management strategies. Agronomy Fact Sheet Series 1993-3, Dept. of Agronomy and Range Science, UC Davis.

Crites, W. 1990. Strategies for dealing with resistant weed plants. Proc. Calif. Weed Conf. 42:145-49.

Haworth, P. 1988. Herbicide resistance in weeds. Proc. Calif. Weed Conf. 40:228-33.

Heap, I. 1999. International survey of herbicide-resistant weeds online. Internet. 04 February 1999. Available at http://www.weedscience.com.

Heap, I. M. 1994. Identification and documentation of herbicide resistance. Phytoprotection 75 (Suppl.).

Holt, J. S. 1992. History of identification of herbicide resistant weeds. Weed Technol. 6:615-20.

Holt, J. S., S. B. Powles, and J. A. M. Holtum. 1993. Mechanisms and agronomic aspects of herbicide resistance. Ann. Rev. Plant Physiol. Plant Molec. Biol. 44:203-29.

Mallory-Smith, C., D. Thill, and D. Morishita. 1993. Herbicide resistant weeds and their management. PNW 437.

Moss, S. R. 1995. Techniques for determining herbicide resistance. Brighton Crop Protection Conference-Weeds. Vol. 2:547-56.

Powles, S. B., and J. A. M. Holtum (eds.). 1994. Herbicide resistance in plants. Biology and Biochemistry. Boca Raton: Lewis Publishers.

Retzinger, E. J., and C. Mallory-Smith. 1997. Classification of herbicides by site of action for weed resistance management strategies. Weed Technol. 11:384-93.

Saari, L. L. 1991. Herbicide resistance in weeds, current status and future perspectives. Proc. Calif. Weed Conf. 43:37-40.

Shaner, D., A. Sinha, and R. Braddock. 1992. Designing strategies to delay development of resistance to herbicides. Proc. First Int. Weed Control Congress. pp 236-39. 


\section{FOR MORE INFORMATION}

You'll find detailed information on many aspects of weed management in these titles and in other publications, slide sets, and videos from UC ANR:

Integrated Pest Management for Tomatoes-Fourth Edition, publication 3274

Integrated Pest Management for Tomatoes, slide set 98/103

Grower's Weed Identification Handbook, publication 4030

Weeds of the West, publication 3350

To order these products, visit our online catalog at http://anrcatalog.ucdavis.edu. You can also place orders by mail, phone, or fax, or request a printed catalog of publications, slide sets, and videos from

University of California

Division of Agriculture and Natural Resources

Communication Services

6701 San Pablo Avenue, 2nd Floor

Oakland, California 94608-1239

Telephone: 1-800-994-8849 or (510) 642-2431, FAX: (510) 643-5470

e-mail inquiries: danrcs@ucdavis.edu

An electronic version of this publication is available on the ANR Communication Services website at http://anrcatalog.ucdavis.edu.

Publication 8012

(C) 2000 by the Regents of the University of California,

Division of Agriculture and Natural Resources. All rights reserved.

The University of California prohibits discrimination against or harassment of any person employed by or seeking employment with the University on the basis of race, color, national origin, religion, sex, physical or mental disability, medical condition (cancer-related or genetic characteristics), ancestry, marital status, age, sexual orientation, citizenship, or status as a covered veteran (special disabled veteran, Vietnam-era veteran or any other veteran who served on active duty during a war or in a campaign or expedition for which a campaign badge has been authorized).

University Policy is intended to be consistent with the provisions of applicable State and Federal laws. Inquiries regarding the University's nondiscrimination policies may be directed to the Affirmative Action/Staff Personnel Services Director, University of California, Agriculture and Natural Resources, 1111 Franklin, 6th Floor, Oakland, CA 94607-5200 (510) 987-0096.

pr-3/00-WJC

ISBN 978-1-60107-192-7 